\title{
Low-Dosed X-Ray Computed Tomography Imaging by Regularized Fully Spatial Fractional-Order Perona-Malik Diffusion
}

\author{
Zhiwu Liao \\ School of Computer Science, Sichuan Normal University, Chengdu, Sichuan 610101, China \\ Correspondence should be addressed to Zhiwu Liao; liaozhiwu@163.com
}

Received 19 September 2013; Accepted 2 November 2013

Academic Editor: Ming Li

Copyright (C) 2013 Zhiwu Liao. This is an open access article distributed under the Creative Commons Attribution License, which permits unrestricted use, distribution, and reproduction in any medium, provided the original work is properly cited.

\begin{abstract}
Existing fractional-order Perona-Malik Diffusion (FOPMD) algorithms used in noise suppressing suffer from undesired artifacts and speckle effect, which hamper FOPMD used in low-dosed X-ray computed tomography (LDCT) imaging. In this paper, we propose a new FOPMD method for low-dose computed tomography (LDCT) imaging, which is called regularized fully spatial FOPMD (RFS-FOPMD), whose numerical scheme is also given based on Grünwald-Letnikov derivative (G-L derivative). Here, fully spatial FOPMD represents all the integer-order derivatives (IODs) in the right hand of Perona-Malik Diffusion (PMD) which are replaced by fractional-order derivatives (FODs). Since the new scheme has advantages of both regularization and FOPMD, it has good abilities in singularities preserving while suppressing noise. Some real sinogram of LDCT are used to compare the different performances not only for some classical but also for some state-of-art diffusion schemes. These schemes include PMD, regularized PMD (RPMD), and FOPMD in (Hu et al. 2012). Experimental results show that besides good ability in edge preserving, the new scheme also has good stability for iteration number and can avoid artifacts and speckle effect with suitable parameters.
\end{abstract}

\section{Introduction}

Perona-Malik diffusion (PMD) proposed in 1990 is a popular technique in image denoising and it is defined as [1]

$$
\frac{\partial u(x, y, t)}{\partial t}=\operatorname{div}[c(\|\nabla u(x, y, t)\|) \nabla u(x, y, t)],
$$

where $u(x, y, 0)$ is the initial gray scale image, $u(x, y, t)$ is the smoothed gray scale image at time $t, \nabla$ denotes the gradient, $\operatorname{div}(\cdot)$ is the divergence operator, and $c(\cdot)$ is the diffusion coefficient.

In 1992, Catté et al. indicated that PMD is ill-posed and they propose a new well-posed method named regularized Perona-Malik diffusion (RPMD), by replacing the gradient $\nabla u$ in diffusion coefficients by the smoothed version $G_{\sigma} \cdot \nabla u$ [2]. Thus, the RPMD can be represented as

$$
\frac{\partial u(x, y, t)}{\partial t}=\operatorname{div}\left[c\left(\left\|G_{\sigma_{1}} \cdot \nabla u(x, y, t)\right\|\right) \nabla u(x, y, t)\right] .
$$

Here $G_{\sigma_{1}}$ is defined as:

$$
G_{\sigma_{1}}=\frac{1}{C} e^{-\left(\left(x^{2}+y^{2}\right) / \sigma_{1}^{2}\right)}
$$

which is a Gaussian function and $C$ is a constant.

In order to eliminate undesired "staircase" of PMD and RPMD, high-order PDEs (typically fourth-order PDEs) for image restoration have been introduced in $[3,4]$. Though these methods can eliminate the staircase effect efficiently, they often leave the image with isolated black and white speckles (so-called speckle effect) [5].

Recently, fractional-order PMD (FOPMD) has been studied in image denoising [5-14], whose fractional order is $\alpha$, $0 \leq \alpha \leq 2$, which is a "natural interpolation" between PMD and fourth-order PDEs. Therefore, it has the benefits of both of PMD and high order PDEs.

Bai and Feng proposed a FSFOD method for image denoising with Euler-Lagrange equations of a cost functional and using Fourier-domain to compute the fractional 
derivative [5]. Jun and Zhihui develop a class of fractionalorder multiscale variational model using G-L definition of fractional-order derivative and propose an efficient condition of the convergence of the model [6].

We also reported an experimental study that used FOPMD for sinogram restoration of low-dosed computed tomography (LDCT) based on fully spatial FOPMD using GL definition [8]. In [8], we observe that FOPMD experiments with different fractional orders show different diffusion behaviors, which leads us to study FOPMD further.

In a most recent study, Hu proposed a new FOPMD by diffusing only on external gradient vector and reported good performance in LDCT imaging [9].

Although above FOPMD have reported on good performance of preserving edges, suppressing staircase and speckle effects, the resulting images of these FOPMD methods still have some artifacts and speckle effect which hamper them to be used in LDCT imaging.

Minimizing the radiation exposure to patients has been one of the major efforts in modern clinical X-ray CT radiology [15-17]. However, the presentation of strong noise degrades the quality of LDCT images dramatically and decreases the accuracy of diagnosis.

Filtering noise from clinical scans is a challenging task, since these scans contain many structures with different shapes, sizes, and contrasts, which should be preserved for making correct diagnosis. In addition, LDCT imaging also requires no artifacts and speckle effect to emerge while denoising because artifacts also increase chances of misdiagnosis. Many strategies have been proposed to reduce noises, but few of them discuss how to avoid artifacts and speckle effect $[8,18-28]$.

The artifacts of existing FOPMD methods used for LDCT imaging are generated by strong noises of LDCT sinogram, which leads to falsely located positions of edges. Thus, the resulting images produce some undesired weak edges, which form the artifacts.

Therefore, one valid method to suppress artifacts and speckle effect of existing FOPMD methods is by smoothing the fractional-order gradient (FOG) in the diffusion coefficients to avoid error-detected edges of the noisy LDCT.

Following the above discussion, we propose a regularized FOPMD, named regularized fully spatial FOPMD (RFSFOPMD), by replacing FOG of diffusion coefficients with its smoothing counterparts while keeping "external" FOD unchanged. Here "fully spatial" represents all the derivatives of right-hand side of PMD equation and "external" indicates the spatial derivatives except for the derivatives used in diffusion coefficients. Since locations of edges can be detected correctly by our new scheme, FOPMD can preserve edges well and avoid artifacts.

The arrangement of this paper is as follows: in Section 2, the EFOGV-PMD is introduced, and then the numerical scheme is given in Section 3, the experiment results are shown and discussed in Section 4, the final part is the conclusions and acknowledgments.

\section{Regularized Fully Spatial Fractional-Order Perona-Malik Diffusion}

In this paper, we use G-L definition defined as follows [29, 30]:

$$
D^{\alpha} g(x)=\lim _{h \rightarrow 0^{+}} \frac{\sum_{k \geq 0}(-1)^{k} C_{k}^{\alpha} g(x-k h)}{h^{\alpha}}, \quad \alpha>0,
$$

where $g(x)$ is a real function, $\alpha>0$ is a real number, $C_{k}^{\alpha}=$ $\Gamma(\alpha+1) /[\Gamma(k+1) \Gamma(\alpha-k+1)]$ is the generalized binomial coefficient, and $\Gamma(\cdot)$ denotes the gamma function.

Isotropic diffusion will damage the image features such as edges, lines, and textures. To avoid the damage, the smoothing has to be adaptively controlled by the amount of smoothing or the direction of smoothing. A classic example of adaptive smoothing is the anisotropic diffusion scheme proposed by Perona and Malik [1], in which the smoothing process is formulated by a partial differential equation (PDE). PMD is formulated in (1).

However, PMD methods suffer from their "staircase" effects. Therefore, FOPMD is proposed to suppress the staircase of PMD.

The fractional-order gradient vector with $\alpha$ order is defined as

$$
\nabla^{\alpha} u(x, y, t)=\left[\nabla_{x}^{\alpha} u(x, y, t), \nabla_{y}^{\alpha} u(x, y, t)\right],
$$

where $\alpha$ is a positive real, $\nabla_{x}^{\alpha} u(x, y, t)$ represents the partial fractional-order derivative of $u(x, y, t)$ with respect to the variable $x$ whose order is $\alpha$, and $\nabla_{y}^{\alpha} u(x, y, t)$ represents the partial fractional-order derivative of $u(x, y, t)$ with respect to the variable $y$ whose order is $\alpha$.

According to [8], FOPMD is defined as

$$
\frac{\partial u(x, y, t)}{\partial t}=\operatorname{div}^{\alpha}\left[c\left(\left\|\nabla^{\alpha} u(x, y, t)\right\|\right) \nabla^{\alpha} u(x, y, t)\right],
$$

where $\operatorname{div}^{\alpha}$ denotes the $\alpha$-order divergence. For the vector $\nabla^{\beta} v=\left[v_{x}^{\beta}, v_{y}^{\beta}\right]$ where $v_{x}^{\beta}, v_{y}^{\beta}$ represent the partial fractionalorder derivative of $u(x, y, t)$ with whose order is $\beta$ respect to the variable $x$ and $y$ respectively, its $\alpha$-order divergence is defined as:

$$
\operatorname{div}^{\alpha} \nabla^{\beta} v=v_{x}^{\beta+\alpha}+v_{y}^{\beta+\alpha} .
$$

However, FOPMD defined by (3) will produce some artifacts for sinogram restoration of LDCT, which increases the probability of error diagnosis. In order to avoid artifacts produced in sinogram restoration of LDCT using FOPMD, we propose a new diffusion model, named regularized fully spatial fractional-order PMD (RFS-FOPMD), where "fully spatial" indicates all derivatives of the right-hand side of (6). That is, the FOD in diffusion coefficient is replaced by its smoothed version.

Therefore, the RFS-FOPMD is given by

$$
\frac{\partial u(x, y, t)}{\partial t}=\operatorname{div}^{\alpha}\left[c\left(\left\|G_{\sigma_{1}} \cdot \nabla^{\alpha} u(x, y, t)\right\|\right) \nabla^{\alpha} u(x, y, t)\right]
$$


with the observed image as the initial condition and $G_{\sigma_{1}}$ is defined in (3).

When $\alpha=1,(6)$ is precisely the PMD and (8) is precisely the RPMD; when $\alpha=2,(6)$ is precisely the fourth-order anisotropic diffusion equation. In this paper, we are interested in $0.5 \leq \alpha \leq 1.5$ since Bai and Feng in [5] suggest that $\alpha=1.5$ in their model has the best performance.

\section{The Numerical Scheme}

An image $U$ will be a 2-dimensional matrix of size $N \times N$. In order to get the aim of anisotropic diffusion along different directions and because the discrete $\alpha$-order gradient $\nabla^{\alpha} u$ is an 8-dimensional vector

$$
\begin{aligned}
& \nabla^{\alpha} \mathbf{u}(\mathbf{i}, \mathbf{j}) \\
& =\left(\nabla_{0}^{\alpha} u(i, j), \nabla_{1}^{\alpha} u(i, j), \nabla_{2}^{\alpha} u(i, j), \nabla_{3}^{\alpha} u(i, j),\right. \\
& \left.\quad \nabla_{4}^{\alpha} u(i, j), \nabla_{5}^{\alpha} u(i, j), \nabla_{6}^{\alpha} u(i, j), \nabla_{7}^{\alpha} u(i, j)\right)^{T},
\end{aligned}
$$

where $T$ represents the transpose of the vector and $\nabla^{\alpha} u_{k}(i, j)$, $k=0, \ldots, 7$ are defined as

$$
\begin{aligned}
& \nabla_{0}^{\alpha} u(i, j)=\sum_{k=0}^{K-1}(-1)^{k} C_{k}^{\alpha} u(i, j+k-(K-1)), \\
& \nabla_{1}^{\alpha} u(i, j)=\sum_{k=0}^{K-1}(-1)^{k} C_{k}^{\alpha} u(i-k+(K-1), j+k-(K-1)), \\
& \nabla_{2}^{\alpha} u(i, j)=\sum_{k=0}^{K-1}(-1)^{k} C_{k}^{\alpha} u(i-k+(K-1), j), \\
& \nabla_{3}^{\alpha} u(i, j)=\sum_{k=0}^{K-1}(-1)^{k} C_{k}^{\alpha} u(i-k+(K-1), j-k+(K-1)), \\
& \nabla_{4}^{\alpha} u(i, j)=\sum_{k=0}^{K-1}(-1)^{k} C_{k}^{\alpha} u(i, j-k+(K-1)), \\
& \nabla_{5}^{\alpha} u(i, j)=\sum_{k=0}^{K-1}(-1)^{k} C_{k}^{\alpha} u(i+k-(K-1), j-k+(K-1)), \\
& \nabla_{7}^{\alpha} u(i, j)=\sum_{k=0}^{\alpha-1}(-1)^{k} C_{k}^{\alpha} u(i+k-(K-1), j+k-(K-1)) .
\end{aligned}
$$

Thus,

$$
\begin{aligned}
\nabla^{2 \alpha} \mathbf{u}(\mathbf{i}, \mathbf{j}) & \\
= & \left(\nabla_{0}^{2 \alpha} u(i, j), \nabla_{1}^{2 \alpha} u(i, j), \nabla_{2}^{2 \alpha} u(i, j), \nabla_{3}^{2 \alpha} u(i, j),\right. \\
& \left.\nabla_{4}^{2 \alpha} u(i, j), \nabla_{5}^{2 \alpha} u(i, j), \nabla_{6}^{2 \alpha} u(i, j), \nabla_{7}^{2 \alpha} u(i, j)\right)^{T},
\end{aligned}
$$

where $T$ represents the transpose of the vector. From (4), we have

$$
\begin{aligned}
& \nabla_{0}^{2 \alpha} u(i, j)=\sum_{k=0}^{K-1}(-1)^{k} C_{k}^{2 \alpha} u(i, j+k-(K-1)), \\
& \nabla_{1}^{2 \alpha} u(i, j)=\sum_{k=0}^{K-1}(-1)^{k} C_{k}^{2 \alpha} u(i-k+(K-1), j+k-(K-1)), \\
& \nabla_{2}^{2 \alpha} u(i, j)=\sum_{k=0}^{K-1}(-1)^{k} C_{k}^{2 \alpha} u(i-k+(K-1), j), \\
& \nabla_{3}^{2 \alpha} u(i, j)=\sum_{k=0}^{K-1}(-1)^{k} C_{k}^{2 \alpha} u(i-k+(K-1), j-k+(K-1)), \\
& \nabla_{4}^{2 \alpha} u(i, j)=\sum_{k=0}^{K-1}(-1)^{k} C_{k}^{2 \alpha} u(i, j-k+(K-1)), \\
& \nabla_{5}^{2 \alpha} u(i, j)=\sum_{k=0}^{K-1}(-1)^{k} C_{k}^{2 \alpha} u(i+k-(K-1), j-k+(K-1)), \\
& \nabla_{7}^{2 \alpha} u(i, j)=\sum_{k=0}^{K-1}(-1)^{k} C_{k}^{2 \alpha} u(i+k-(K-1), j), \\
& \nabla_{k=0}^{K-1}(-1)^{k} C_{k}^{2 \alpha} u(i+k-(K-1), j+k-(K-1)) .
\end{aligned}
$$

Let

$$
\mathbf{g}=\left(g_{0}, g_{1}, g_{2}, g_{3}, g_{4}, g_{5}, g_{6}, g_{7}\right)^{T}
$$

where $T$ represents the transpose of the vector and $g_{k}, k=$ $0, \ldots, 7$ are defined as

$$
g_{k}=\frac{g\left(\left\|G_{\sigma_{1}} \cdot \nabla_{k}^{\alpha} u(i, j)\right\|\right)}{\sum_{n=0}^{7} g\left(\left\|G_{\sigma_{1}} \cdot \nabla_{n}^{\alpha} u(i, j)\right\|\right)}, \quad k=0,1, \ldots, 7,
$$

where $\nabla_{k}^{\alpha} u(i, j), k=0, \ldots 7$ defined in (9) are the components of vector $\nabla^{\alpha} \mathbf{u}(\mathbf{i}, \mathbf{j})$ and $\sum_{n=0}^{7} g\left(\left\|G_{\sigma_{1}} \cdot \nabla_{n}^{\alpha} u(i, j)\right\|\right)$ is the normalized constant, $g$ is the decreasing function of absolute value of $\nabla_{k}^{\alpha} u(i, j), k=0, \ldots 7$, and $g\left(\left\|\nabla_{k}^{\alpha} u(x, y, t)\right\|\right)$ is defined as

$$
g\left(\left\|\nabla_{k}^{\alpha} u(x, y, t)\right\|\right)=e^{-\left(\left\|\nabla_{k}^{\alpha} u(x, y, t)\right\| / \sigma\right)^{2}}, \quad k=0, \ldots, 7
$$

or

$$
\begin{array}{r}
g\left(\left\|\nabla_{k}^{\alpha} u(x, y, t)\right\|\right)=\frac{1}{1+\left(\left\|\nabla_{k}^{\alpha} u(x, y, t)\right\| / \sigma\right)^{2}}, \\
k=0, \ldots, 7,
\end{array}
$$

where $\|\cdot\|$ is the module of the fractional-order vector and the constant $\sigma$ controls the sensitivity to edges. 
The new FOPMD based on G-L fractional-order derivative is defined as

$$
\frac{\partial u(i, j, t)}{\partial t}=\operatorname{div}^{\alpha}\left(\begin{array}{c}
g_{0} \nabla_{0}^{\alpha} u(i, j, t) \\
g_{1} \nabla_{1}^{\alpha} u(i, j, t) \\
g_{2} \nabla_{2}^{\alpha} u(i, j, t) \\
g_{3} \nabla_{3}^{\alpha} u(i, j, t) \\
g_{4} \nabla_{4}^{\alpha} u(i, j, t) \\
g_{5} \nabla_{5}^{\alpha} u(i, j, t) \\
g_{6} \nabla_{6}^{\alpha} u(i, j, t) \\
g_{7} \nabla_{7}^{\alpha} u(i, j, t)
\end{array}\right),
$$

where $g_{k}, k=0, \ldots, 7$ defined in (14) are the components of $\mathrm{g}$ in (13).

The above equation can be represented as

$$
\frac{\partial u(i, j, t)}{\partial t}=\sum_{k=0}^{7} g_{k} \nabla_{k}^{2 \alpha} u(i, j, t)
$$

where $\sum_{k=0}^{7} g_{k}=1$ and $\nabla_{k}^{2 \alpha} u(i, j, t)$ can be computed according to (8).

Thus, the explicit form for solving (18) is

$$
u(i, j, t+1)=u(i, j, t)+\lambda \sum_{k=0}^{7} g_{k} \nabla_{k}^{2 \alpha} u(i, j, t),
$$

where $u(i, j, t+1)$ is the gray level of $(i, j)$ at time $t+1$ and $\lambda$ is the integration constant $(0 \leq \lambda \leq 1 / 7)$.

To summarize, our sinogram restoration approach is done in the following steps:

(1) let the input sinogram be $U$ and set $t=1, U_{t}=U$, input iterative numbers $n$, Gaussian deviations $\sigma_{1}$ of regularized Gaussian kernel in (3), fractional order $\alpha$, integration constant $\lambda$ in (19), gradient modulus threshold $\sigma$ that controls the conduction used in (15) or (16), and choose (15) or (16) as diffusion coefficients;

(2) compute $\alpha$-order gradient vector $\nabla^{\alpha} \mathbf{u}$ using (9);

(3) compute diffusion coefficients vector $\mathbf{g}$ using (13)(16);

(4) compute $2 \alpha$-order gradient vector $\nabla^{2 \alpha} \mathbf{u}$ using (11);

(5) compute $U_{t+1}$ using (19), and set $t=t+1$, if $t=n$, output sinogram $U_{t}$; else goto step 2;

(6) return back-project sinogram $U_{t}$ into the image $I$.

\section{Experiments and Discussion}

The main objective for LDCT imaging is to delete the noise and avoid artifacts while preserving anatomy details for the back-projection images.
Two abdominal CT images of a 58-year-old man and two abdominal CT images of a 62-year-old woman with different doses were scanned from a 16-multi detector-row CT unit (Somatom Sensation 16; Siemens Medical Solutions) using $120 \mathrm{kVp}$ and $5 \mathrm{~mm}$ slice thickness. Other remaining scanning parameters are gantry rotation time, 0.5 second; detector configuration (number of detector rows section thickness), $16 \times 1.5 \mathrm{~mm}$; table feed per gantry rotation, $24 \mathrm{~mm}$; pitch, $1: 1$, and reconstruction method, back projection (FBP) algorithm with the soft-tissue convolution kernel "B30f." Different CT doses were controlled by using two different fixed tube currents $60 \mathrm{mAs}$ and $150 \mathrm{mAs}(60 \mathrm{~mA}$ or $150 \mathrm{mAs})$ for LDCT and standard-dose CT (SDCT) protocols, resp.). The CT dose index volume (CTDIvol) for LDCT images and SDCT images is in positive linear correlation to the tube current and is calculated to be approximately ranging between $15.32 \mathrm{mGy}$ and 3.16 mGy [28] (see Figures 1(a)-1(d)).

In order to compare our method with classical PM and other state-of-art FOPMD methods, three compared methods: PMD [1], regularized PMD (RPM) [2], and FOPMD are proposed in [8]. According to the numerical scheme of PMD and RPMD, they used half-point central difference discretization scheme, while FOPMD in [8] and RFS-FOPMD use integer-point unilateral difference discretization scheme.

In order to ensure that the comparison is put on a fair level, the common used parameters are set to the same value. The common used parameters for four methods include gradient modulus threshold $\sigma$ that controls the conduction, integration constant $\lambda$, and iteration number $t$. Due to numerical stability, $\lambda$ is set to its maximum value $1 / 100$ and $\sigma$ is set to 30 to reduce iteration number.

The iteration number $t$ is very important in all comparison methods. That is, big $t$ will make smooth image while small $t$ will still leave a lot of noise. In order to study the performance of four compared methods with different iteration numbers $t$ and other fixed parameters, $t$ is set to 20 , 50 , and 100 , respectively.

The standard deviation of smoothed Gaussian kernel for the image $\sigma_{1}$ used for RPMD is set to 1 since, in [2], the authors suggest that $\sigma_{1}$ should be a small number.

On sinogram space, FOPMD with $\alpha=0.5, \alpha=0.8$ and $\alpha=1.2$ is carried on two image collections.

Since bigger iteration number leads to smoother denoised results sometimes, it also leads to dark processed images by posing too big integration constant $\lambda$. In order to observe the behaviors of big iteration number clearly, Figure 1 sets iteration number $t=100$. Comparing all the original SDCT images in Figures 1(a) and 1(c), LDCT images Figures 1(b) and $1(\mathrm{~d})$ were severely degraded by nonstationary noise. All denoised images in Figure 1 can suppress most of noises. Particular, FOPMD and RFS-FOPMD can provide very satisfied images with little noise and preserving all useful anatomy structures. However, denoised images of PMD and RPMD are oversmooth, which lost a lot of details.

In order to test the consistency of the definitions of different integer order or fractional order, we set fractionalorder $\alpha=1$, in which a two fractional-order PMD should have same forms and they also correspond to the order of PMD and RPMD (see Figures 1(e)-1(1)). Observing Figures 


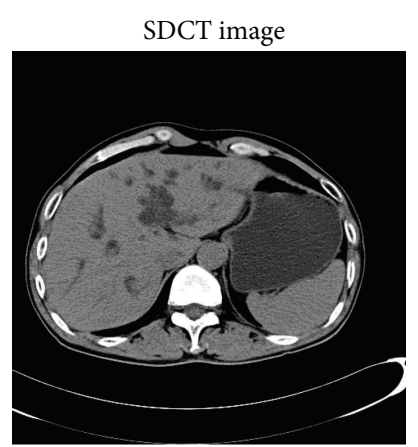

(a) Original SDCT image with tube current time product $150 \mathrm{mAs}$

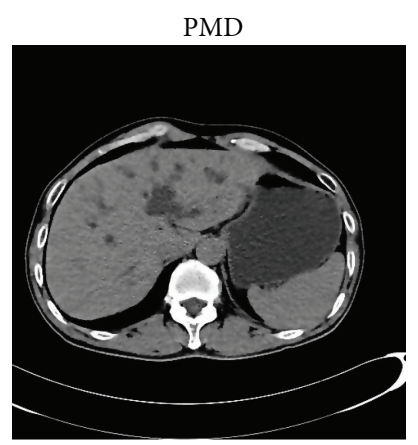

(e) LDCT image (b) processed by PMD with $\sigma=30, \lambda=1 / 100$ and iteration number is 100

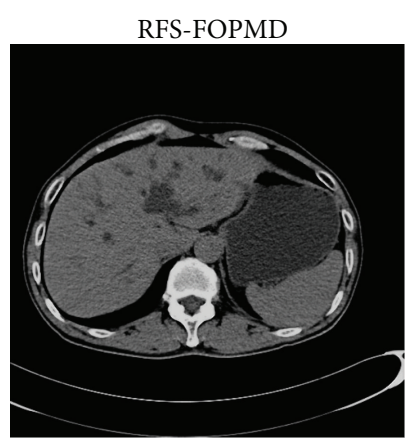

(i) LDCT image (b) processed by RFS-FOPMD with $\sigma=30, \alpha=1$, $\sigma_{1}=1$, and $\lambda=1 / 100$ and iteration number is 100

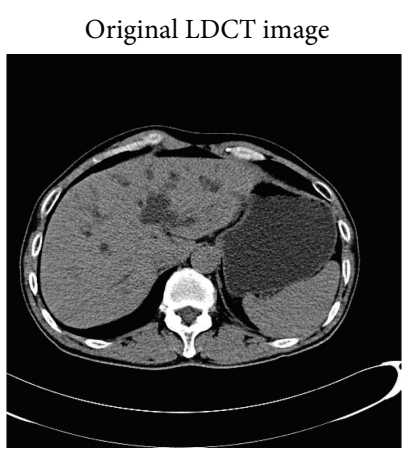

(b) Original LDCT image with tube current time product $30 \mathrm{mAs}$

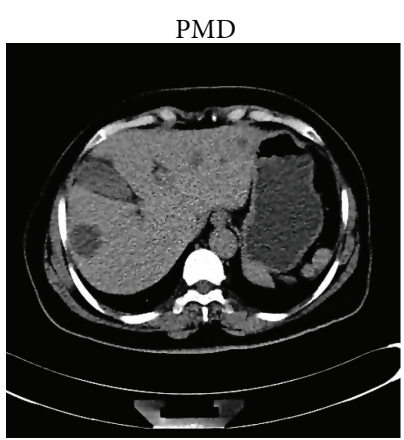

(f) LDCT image (d) processed by PMD with $\sigma=30, \lambda=1 / 100$ and iteration number is 100

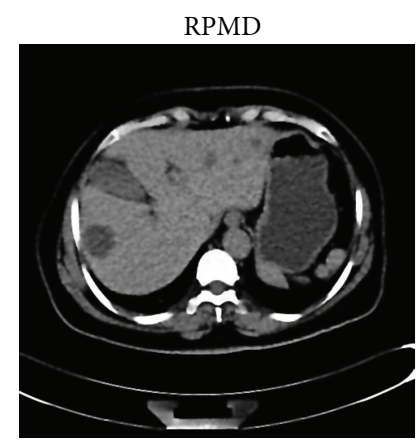

(j) LDCT image (d) processed by RPMD with $\sigma=30, \sigma_{1}=1$, and $\lambda=1 / 100$ and iteration number is 100

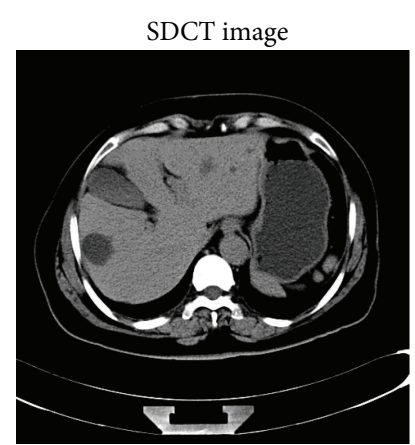

(c) Original SDCT image with tube current time product $150 \mathrm{mAs}$

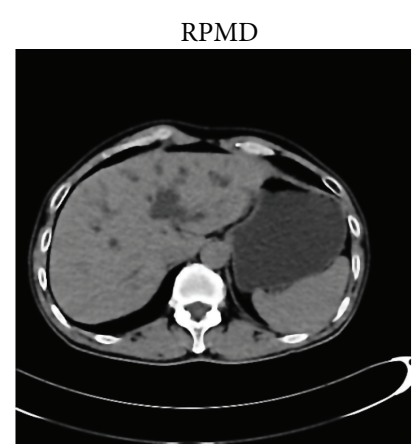

(g) LDCT image (b) processed by RPMD with $\sigma=30, \sigma_{1}=1, \lambda=$ $1 / 100$ and iteration number is 100

FOPMD

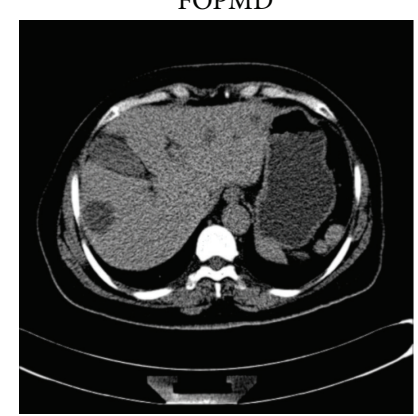

(k) LDCT image (d) processed by FOPMD in [8] with $\sigma=30, \alpha=$ 1 , and $\lambda=1 / 100$ and iteration number is 100

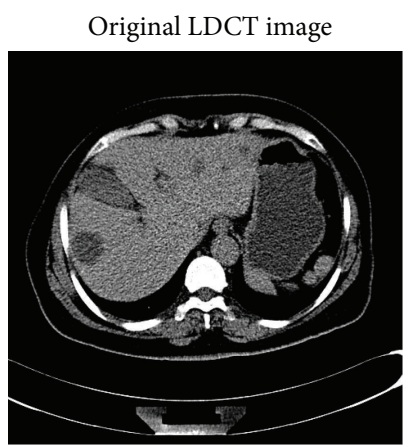

(d) Original LDCT image with tube current time product $60 \mathrm{mAs}$

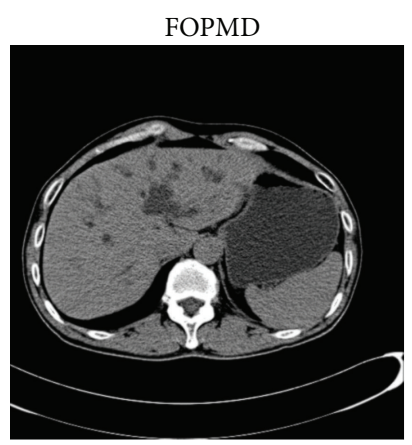

(h) LDCT image (b) processed by FOPMD in [8] with $\sigma=30, \alpha=$ 1 , and $\lambda=1 / 100$ and iteration number is 100

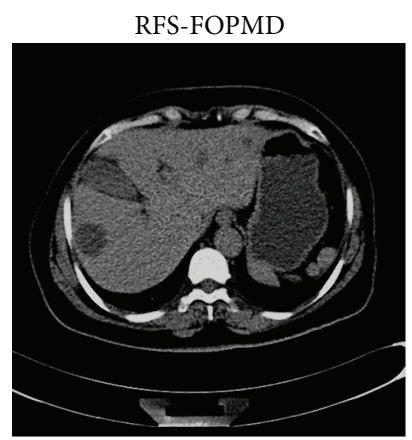

(l) LDCT image (d) processed by RFS-FOPMD with $\sigma=30, \sigma_{1}=1$, $\alpha=1$, and $\lambda=1 / 100$ and iteration number is 100

FIGURE 1: Original SDCT and LDCT images ((a)-(d)), LDCT images processed by PMD, RPMD, FOPMD, and RFS-FOPMD with fractional order $\alpha=1$ and iteration number is set to 100 .

1(h) and $1(\mathrm{i}), 1(\mathrm{k})$ and $1(\mathrm{l})$, we can find that the denoised images are identical, which demonstrate that the fractionalorder definitions between [8] and RFS-FOPMD are identical when $\alpha=1$.

However, the resulting images of PMD and RPMD are quite different to the images denoised by FOPMD and RFSFOPMD. That is, the images processed by PMD and RPMD are smoother than the images processed by FOPMD and RFS-FOPMD. Just as introduced in the previous paragraph, different discretization schemes lead to this interesting result.
Since two FOMD schemes provide more satisfied results, we only compare two FOPMD methods with different fractional orders (see Figure 2) and different iteration numbers (see Figure 3).

In order to compare denoised results of two FOPMD schemes with different fractional orders, two original LDCT images in Figures 1(b) and 1(d) are used with iteration number $t=100$ and fractional-order $\alpha=0.5, \alpha=0.8$, and $\alpha=1.2$. From the second and the fourth rows of Figure 2, we can conclude that the resulting images of RFS-FOPMD are very 
FOPMD

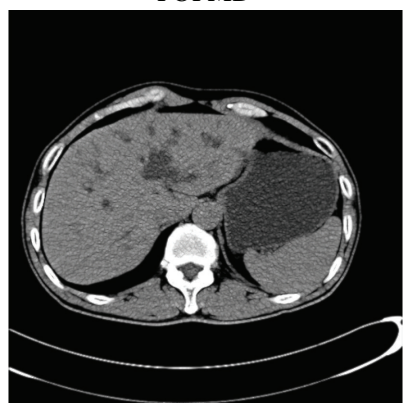

(a) LDCT image Figure 1(b) processed by FOPMD in [8] with $\sigma=$ $30, \alpha=0.5$, and $\lambda=1 / 100$ and iteration number is 100

RFS-FOPMD

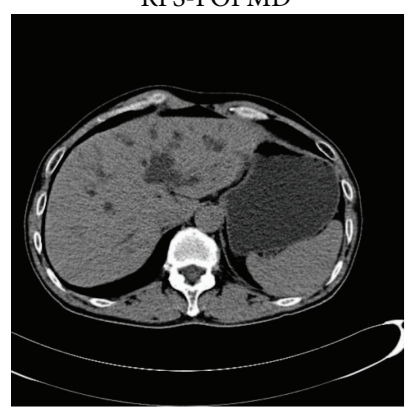

(e) LDCT image Figure 1(b) processed by RFS-FOPMD with $\sigma=30$, $\sigma_{1}=1, \alpha=0.8$, and $\lambda=1 / 100$ and iteration number is 100

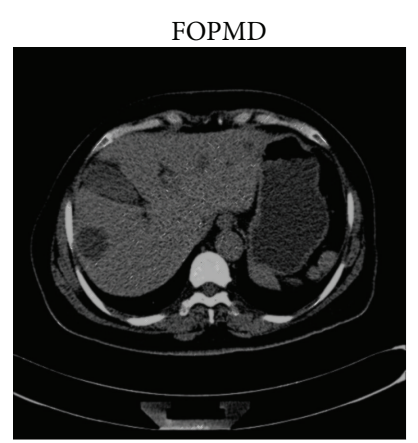

(i) LDCT image Figure 1(d) processed by FOPMD in [8] with $\sigma=$ $30, \alpha=1.2$, and $\lambda=1 / 100$ and iteration number is 100
FOPMD

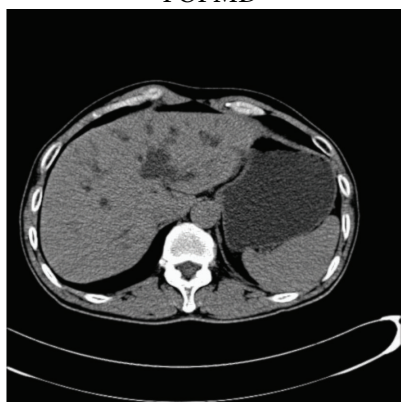

(b) LDCT image Figure 1(b) processed by FOPMD in [8] with $\sigma=$ $30, \alpha=0.8$, and $\lambda=1 / 100$ and iteration number is 100

RFS-FOPMD

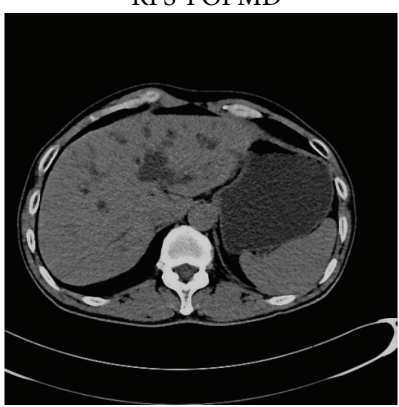

(f) LDCT image Figure 1(b) processed by RFS-FOPMD with $\sigma=30$, $\sigma_{1}=1, \alpha=1.2$, and $\lambda=1 / 100$ and iteration number is 100

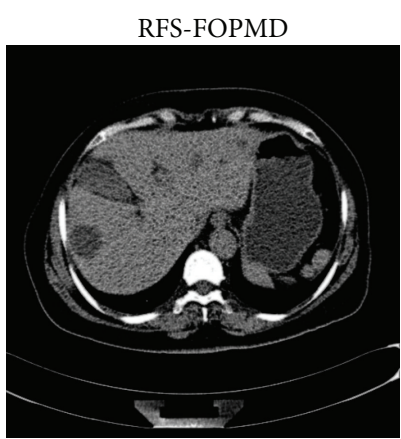

(j) LDCT image Figure 1(d) processed by RFS-FOPMD with $\sigma=30$, $\sigma_{1}=1, \alpha=0.5$, and $\lambda=1 / 100$ and iteration number is 100

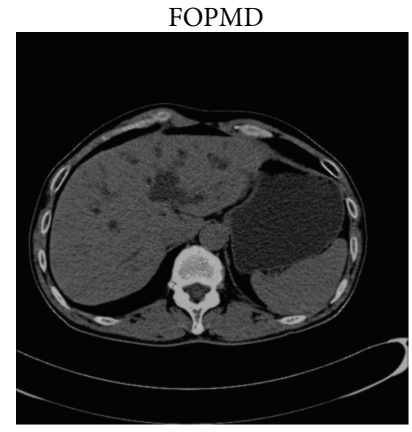

(c) LDCT image Figure 1(b) processed by FOPMD in [8] with $\sigma=$ $30, \alpha=1.2$, and $\lambda=1 / 100$ and iteration number is 100

FOPMD

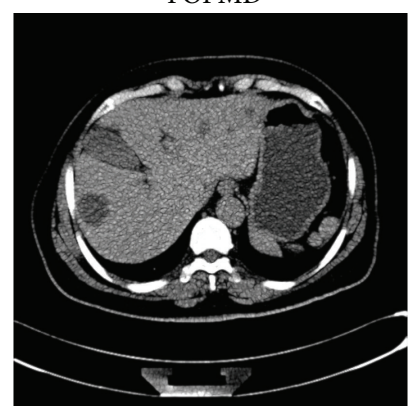

(g) LDCT image Figure 1(d) processed by FOPMD in [8] with $\sigma=$ $30, \alpha=0.5$, and $\lambda=1 / 100$ and iteration number is 100

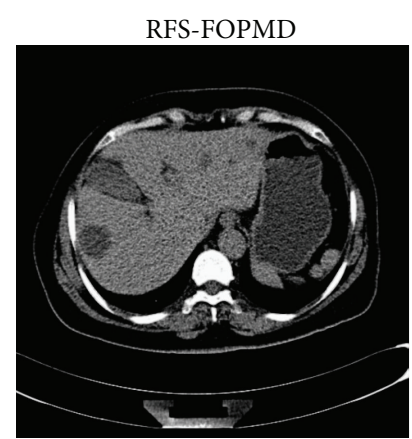

(k) LDCT image Figure 1(d) processed by RFS-FOPMD with $\sigma=30$, $\sigma_{1}=1, \alpha=0.8$, and $\lambda=1 / 7$ and iteration number is 15

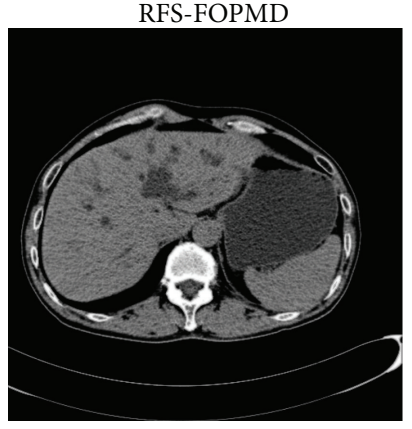

(d) LDCT image Figure 1(b) processed by RFS-FOPMD with $\sigma=30$, $\sigma_{1}=1, \alpha=0.5$, and $\lambda=1 / 100$ and iteration number is 100

FOPMD

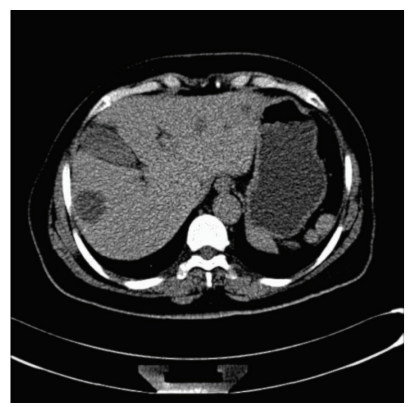

(h) LDCT image Figure 1(d) processed by FOPMD in [8] with $\sigma=$ $30, \alpha=0.8$, and $\lambda=1 / 100$ and iteration number is 100

RFS-FOPMD

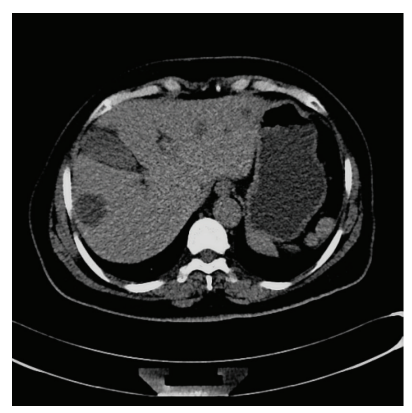

(l) LDCT image Figure 1(d) processed by RFS-FOPMD with $\sigma=$ $30, \sigma_{1}=1, \alpha=1.2$, and $\lambda=1 / 100$ and iteration number is 100

FIGURE 2: LDCT images (Figures 1(b) and 1(d)) processed by FOPMD and RFS-FOPMD with different fractional orders and iteration number is set to 100. The first column: $\alpha=0.5$; the second column: $\alpha=0.8$, and the third column: $\alpha=1.2$. The first row: Figure $1(\mathrm{~b})$ processed by FOPMD; the second row: Figure 1(b) processed by RFS-FOPMD; the third row: Figure 1(d) processed by FOPMD, and the fourth row: Figure $1(\mathrm{~d})$ processed by RFS-FOPMD.

satisfied and they become smoother when $\alpha$ becomes bigger, which is coherent with our intuition, for example, bigger fractional-order smoother resulting images.

However, denoised images in Figures 2(a) and 2(g) with $\alpha=0.5$ using FOPMD in [8] have many artifacts, which are small black circles in two images. Although big fractionalorder FOPMD proposed in [8] will decrease the artifacts, its denoised images in Figures 2(c) and 2(i) are very dark comparing with the original LDCT images in Figures 1(b) and 1(d). Images in Figures 2(c) and 2(i) also have some isolated artificial white points, which are called speckle effect. It is obvious that resulting images in Figures 2(b) and 2(h) with $\alpha=0.8$ processed by FOPMD in [8] have the best performance in three image series with different $\alpha$. 
FOPMD

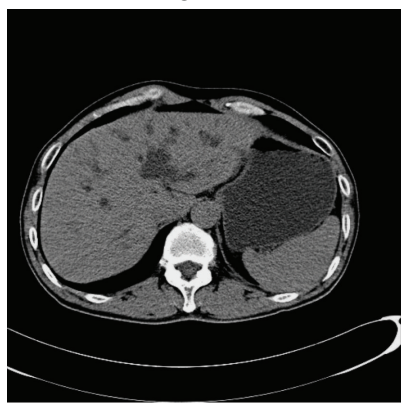

(a) LDCT image (Figure 1(b)) processed by FOPMD in [8] with $\sigma=$ $30, \alpha=1.2$, and $\lambda=1 / 100$ and iteration number is 20

RFS-FOPMD

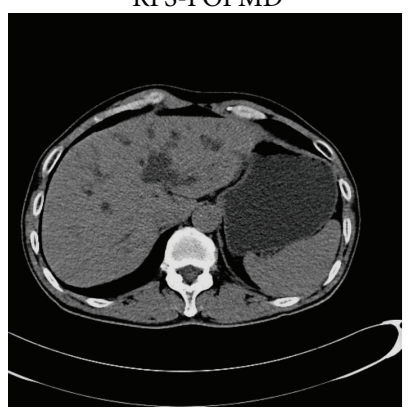

(e) LDCT image (Figure 1(b)) processed by RFS-FOPMD with $\sigma=30$, $\sigma_{1}=1, \alpha=1.2$, and $\lambda=1 / 100$ and iteration number is 50

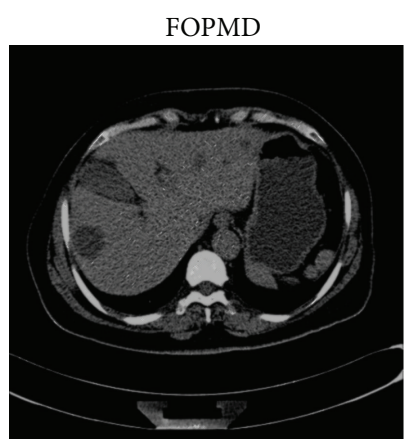

(i) LDCT image (Figure 1(d)) processed by FOPMD in [8] with $\sigma=$ $30, \alpha=1.2$, and $\lambda=1 / 100$ and iteration number is 100
FOPMD

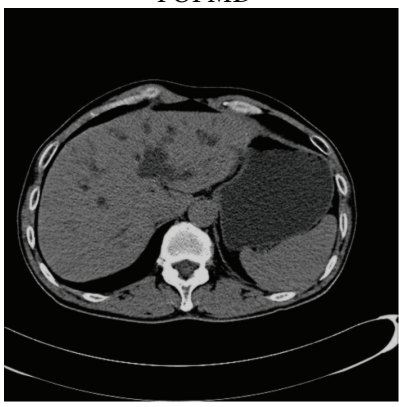

(b) LDCT image (Figure 1(b)) processed by FOPMD in [8] with $\sigma=$ $30, \alpha=1.2$, and $\lambda=1 / 100$ and iteration number is 50

RFS-FOPMD

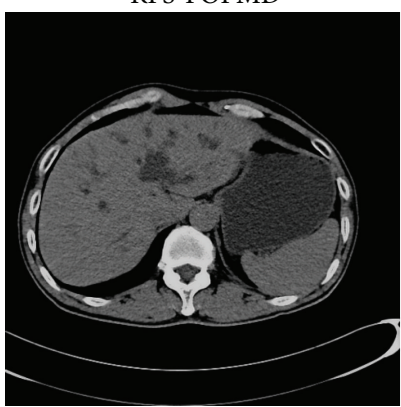

(f) LDCT image (Figure 1(b)) processed by RFS-FOPMD with $\sigma=30$, $\sigma_{1}=1, \alpha=1.2$, and $\lambda=1 / 100$ and iteration number is 100

RFS-FOPMD

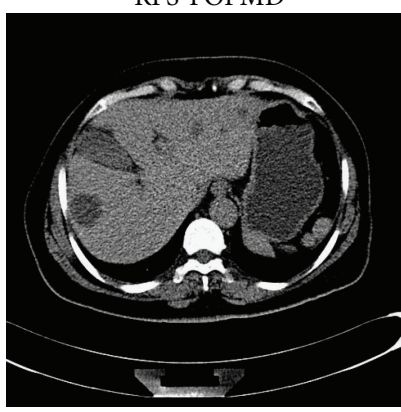

(j) LDCT image (Figure 1(d)) processed by RFS-FOPMD with $\sigma=30$ $\alpha=1.2, \sigma_{1}=1$, and $\lambda=1 / 100$ and iteration number is 20
FOPMD

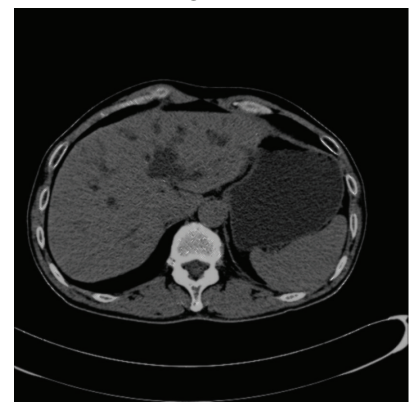

(c) LDCT image (Figure 1(b)) processed by FOPMD in [8] with $\sigma=$ $30, \alpha=1.2$, and $\lambda=1 / 100$ and iteration number is 100

FOPMD

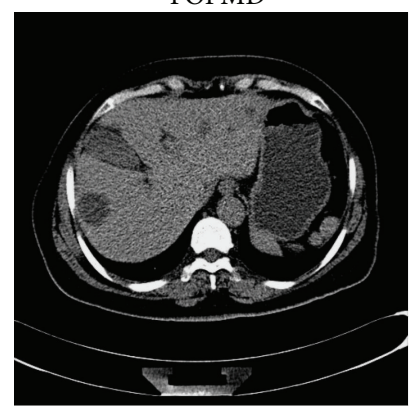

(g) LDCT image (Figure 1(d)) processed by FOPMD in [8] with $\sigma=$ $30, \alpha=1.2$, and $\lambda=1 / 100$ and iteration number is 20

RFS-FOPMD

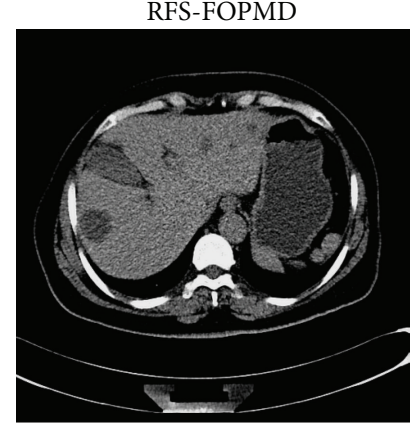

(k) LDCT image (Figure 1(d)) processed by RFS-FOPMD with $\sigma=30$ $\alpha=1.2, \sigma_{1}=1$, and $\lambda=1 / 100$ and iteration number is 50

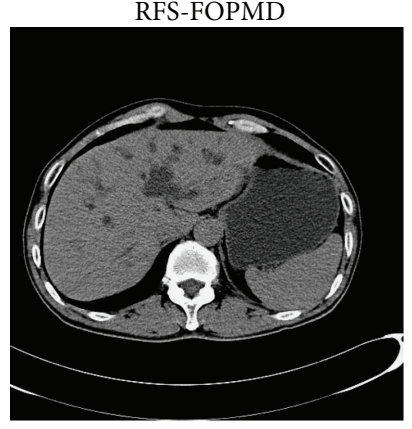

(d) LDCT image (Figure 1(b)) processed by RFS-FOPMD with $\sigma=30$, $\sigma_{1}=1, \alpha=1.2$, and $\lambda=1 / 100$ and iteration number is 20

FOPMD

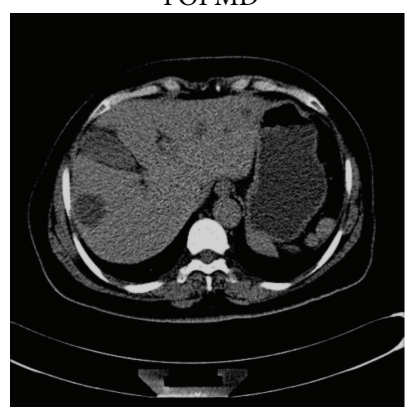

(h) LDCT image (Figure 1(d)) processed by FOPMD in [8] with $\sigma=$ $30, \alpha=1.2$, and $\lambda=1 / 100$ and iteration number is 50

RFS-FOPMD

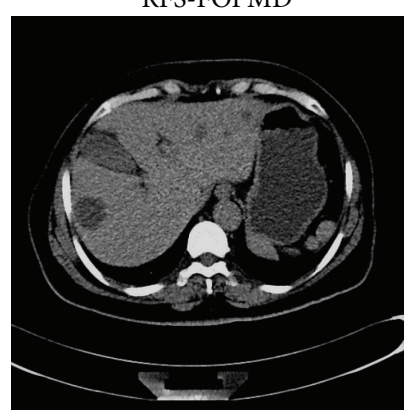

(l) LDCT image (Figure 1(d)) processed by RFS-FOPMD with $\sigma=$ $30, \alpha=1.2, \sigma_{1}=1$, and $\lambda=1 / 100$ and iteration number is 100

FIGURE 3: LDCT images (Figures 1(b) and 1(d)) processed by FOPMD and RFS-FOPMD with different iteration numbers and the fractionalorder $\alpha=1.2$. The first column: iteration number is 20; the second column: iteration number is 50 and the third column: iteration number is 100. The first row: Figure 1(b) processed by FOPMD; the second row: Figure 1(b) processed by RFS-FOPMD; the third row: Figure 1(d) processed by FOPMD and the fourth row: Figure 1(d) processed by RFS-FOPMD.

Generally, artifacts in denoised images are oscillations near edges, caused by that the low-passed filtering is not processed correctly near the real edges. That is, some smooth regions near edges are regarded falsely as edges, which makes these error edges preserved. Therefore, improving accuracy of edge detection is a good choice for improving the performance of FOPMD in [8]. In this paper, we use regularization for FOPMD in [8] to locate edges correctly.
Intuitively, processed images with bigger iteration number correspond to smoother images. In order to check the influence of iteration numbers for FOPMD in [8] and RFS-FOPMD, processed image series of two original LDCT images, Figures 1(b) and 1(d) with different iteration numbers 20, 50, and 100 are shown in Figure 3.

The resulting images in the first and the third rows of Figure 3 are the processed images using FOPMD in [8]. 
Comparing with original LDCT images in Figures 1(b) and $1(\mathrm{~d})$, all resulting images with different iteration numbers have less noise. In addition, the smoother images can be obtained as the iteration number becomes bigger. However, the most undesired default for FOPMD in [8] is that resulting images become dark as the iteration number becomes big. Moreover, except for Figure 3(g), the resulting images in the third row have some isolated white points, which are the speckle effect.

The resulting images in the second and the fourth rows of Figure 3 show that RFS-FOPMD with different iteration numbers is very satisfied and it becomes smoother when the iteration number becomes bigger, which is coherent with our intuition. Another attractive nature for RFS-FOPMD about iteration is that the smoothing shown in these images is very slow. That is, the resulting images Figures $3(\mathrm{~d})$ and $3(\mathrm{j})$ with iteration number 20 are slightly different to the images Figures 3(f) and 3(l) with iteration number 100. This nature shows that RFS-FOPMD has good stability. Therefore, it is not sensitive to iteration number.

All existing FOPMD methods at least suffer from speckle effect from the resulting images of these images. Fortunately, RFS-FOPMD can avoid artifacts, dark images, and speckle effect partly, which ensure its applications in sinogram restoration. More important for the new scheme is its stability, which makes it not sensitive to the iteration number.

\section{Conclusions}

In this paper, we propose a new FOPMD, RFS-FOPMD, for LDCT sinogram imaging based on G-L fractional-order derivative definition. RFS-FOPMD not only has good ability in preserving edges while denoising, but it also can avoid artifacts, dark images, and speckle effects of FOPMD in [8] and other existing FOPMD schemes partly by improving the performance of edges locating by regularization, which ensures that RFS-FOPMD can be used for sinogram restoration of LDCT. Of more importance, RFS-FOPMD has good stability for iteration numbers, which makes it not sensitive to the iteration number choice.

\section{Acknowledgments}

This paper is supported by the National Natural Science Foundation of China (no. 60873102), Major State Basic Research Development Program (no. 2010CB732501), and Open Foundation of Visual Computing and Virtual Reality Key Laboratory of Sichuan Province (no. J2010N03). This work was supported by a Grant from the National High Technology Research and Development Program of China (no. 2009AA12Z140).

\section{References}

[1] P. Perona and J. Malik, "Scale-space and edge detection using anisotropic diffusion," IEEE Transactions on Pattern Analysis and Machine Intelligence, vol. 12, no. 7, pp. 629-639, 1990.

[2] F. Catté, P.-L. Lions, J.-M. Morel, and T. Coll, "Image selective smoothing and edge detection by nonlinear diffusion," SIAM Journal on Numerical Analysis, vol. 29, no. 1, pp. 182-193, 1992.
[3] Y.-L. You and M. Kaveh, "Fourth-order partial differential equations for noise removal," IEEE Transactions on Image Processing, vol. 9, no. 10, pp. 1723-1730, 2000.

[4] T. Chan, A. Marquina, and P. Mulet, "High-order total variation-based image restoration," SIAM Journal on Scientific Computing, vol. 22, no. 2, pp. 503-516, 2000.

[5] J. Bai and X.-C. Feng, "Fractional-order anisotropic diffusion for image denoising," IEEE Transactions on Image Processing, vol. 16, no. 10, pp. 2492-2502, 2007.

[6] Z. Jun and W. Zhihui, "A class of fractional-order multi-scale variational models and alternating projection algorithm for image denoising," Applied Mathematical Modelling, vol. 35, no. 5, pp. 2516-2528, 2011.

[7] M. Janev, S. Pilipović, T. Atanacković, R. Obradović, and N. Ralević, "Fully fractional anisotropic diffusion for image denoising," Mathematical and Computer Modelling, vol. 54, no. 1-2, pp. 729-741, 2011.

[8] S. Hu, Z. Liao, and W. Chen, "Sinogram restoration for lowdosed X-ray computed tomography using fractional-order Perona-Malik diffusion," Mathematical Problems in Engineering, vol. 2012, Article ID 391050, 13 pages, 2012.

[9] S. Hu, "External fractional-order gradient vector Perona-Malik diffusion for sinogram restoration of low-dosed X-ray computed tomography," Advances in Mathematical Physics, vol. 2013, Article ID 516919, 14 pages, 2013.

[10] C. Cattani, A. Ciancio, and B. Lods, "On a mathematical model of immune competition," Applied Mathematics Letters, vol. 19, no. 7, pp. 678-683, 2006.

[11] C. Cattani and A. Ciancio, "Separable transition density in the hybrid model for tumor-immune system competition," Computational and Mathematical Methods in Medicine, vol. 2012, Article ID 610124, 6 pages, 2012.

[12] M. Li, Y. Q. Chen, J. Y. Li, and W. Zhao, "Hölder scales of sea level," Mathematical Problems in Engineering, vol. 2012, Article ID 863707, 22 pages, 2012.

[13] M. Li, W. Zhao, and C. Cattani, "Delay bound: fractal traffic passes through servers," Mathematical Problems in Engineering, vol. 2013, Article ID 157636, 15 pages, 2013.

[14] M. Li and W. Zhao, "On 1/f noise," Mathematical Problems in Engineering, vol. 2012, Article ID 673648, 23 pages, 2012.

[15] D. J. Brenner and E. J. Hall, "Computed tomography-an increasing source of radiation exposure," The New England Journal of Medicine, vol. 357, no. 22, pp. 2277-2284, 2007.

[16] L. Yu, "Radiation dose reduction in computed tomography: techniques and future perspective," Imaging in Medicine, vol. 1, no. 1, pp. 65-84, 2009.

[17] J. Weidemann, G. Stamm, M. Galanski, and M. Keberle, "Comparison of the image quality of various fixed and dose modulated protocols for soft tissue neck CT on a GE Lightspeed scanner," European Journal of Radiology, vol. 69, no. 3, pp. 473477, 2009.

[18] M. K. Kalra, M. M. Maher, M. A. Blake et al., "Detection and characterization of lesions on low-radiation-dose abdominal CT images postprocessed with noise reduction filters," Radiology, vol. 232, no. 3, pp. 791-797, 2004.

[19] J. C. Giraldo, Z. S. Kelm, L. S. Guimaraes et al., "Comparative study of two image space noise reduction methods for computed tomography: bilateral filter and nonlocal means," in Proceedings of the Conference Proceedings IEEE Engineering in Medicine and Biology Society, vol. 1, pp. 3529-3532, 2009. 
[20] A. Manduca, L. Yu, J. D. Trzasko et al., "Projection space denoising with bilateral filtering and CT noise modeling for dose reduction in CT,' Medical Physics, vol. 36, no. 11, pp. 49114919, 2009.

[21] N. Mail, D. J. Moseley, J. H. Siewerdsen, and D. A. Jaffray, “The influence of bowtie filtration on cone-beam CT image quality," Medical Physics, vol. 36, no. 1, pp. 22-32, 2009.

[22] Z. Liao, S. Hu, and W. Chen, "Determining neighborhoods of image pixels automatically for adaptive image denoising using nonlinear time series analysis," Mathematical Problems in Engineering, vol. 2010, Article ID 914564, 14 pages, 2010.

[23] H. Lu, I.-T. Hsiao, X. Li, and Z. Liang, "Noise properties of low-dose CT projections and noise treatment by scale transformations," in Proceedings of the IEEE Nuclear Science Symposium Conference Record, pp. 1662-1666, November 2001.

[24] J. Xu and B. M. W. Tsui, "Electronic noise modeling in statistical iterative reconstruction," IEEE Transactions on Image Processing, vol. 18, no. 6, pp. 1228-1238, 2009.

[25] I. A. Elbakri and J. A. Fessler, "Statistical image reconstruction for polyenergetic X-ray computed tomography," IEEE Transactions on Medical Imaging, vol. 21, no. 2, pp. 89-99, 2002.

[26] J. Wang, H. Lu, J. Wen, and Z. Liang, "Multiscale penalized weighted least-squares sinogram restoration for low-dose Xray computed tomography," IEEE Transactions on Biomedical Engineering, vol. 55, no. 3, pp. 1022-1031, 2008.

[27] J. Wang, T. Li, H. Lu, and Z. Liang, "Penalized weighted least-squares approach to sinogram noise reduction and image reconstruction for low-dose X-ray computed tomography," IEEE Transactions on Medical Imaging, vol. 25, no. 10, pp. 12721283, 2006.

[28] Y. Chen, W. Chen, X. Yin et al., "Improving low-dose abdominal CT images by weighted intensity averaging over large-scale neighborhoods," European Journal of Radiology, vol. 80, no. 2, pp. e42-e49, 2011.

[29] M. D. Ortigueira and F. Coito, "From differences to derivatives," Fractional Calculus and Applied Analysis, vol. 7, no. 4, pp. 459471, 2004.

[30] K. B. Oldham and J. Spanier, The Fractional Calculus, Academic Press, New York, NY, USA, 1974. 


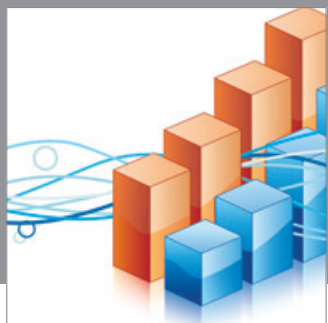

Advances in

Operations Research

mansans

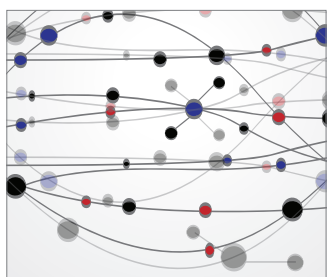

The Scientific World Journal
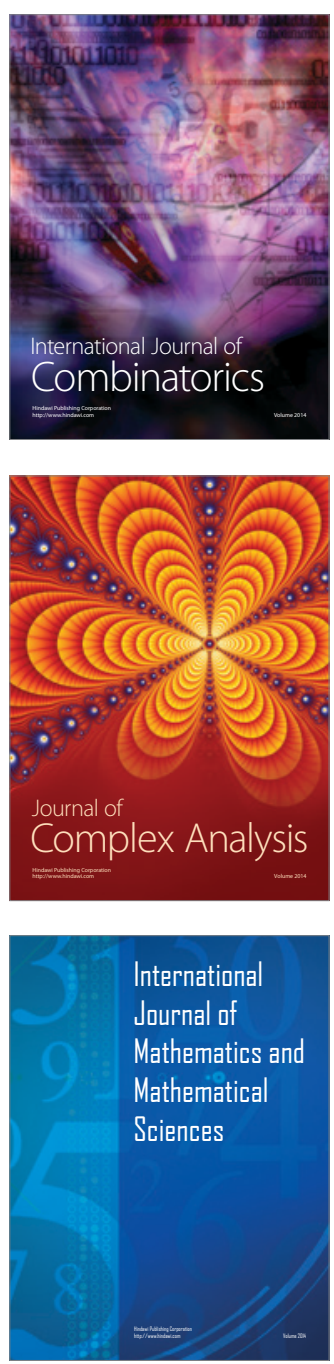
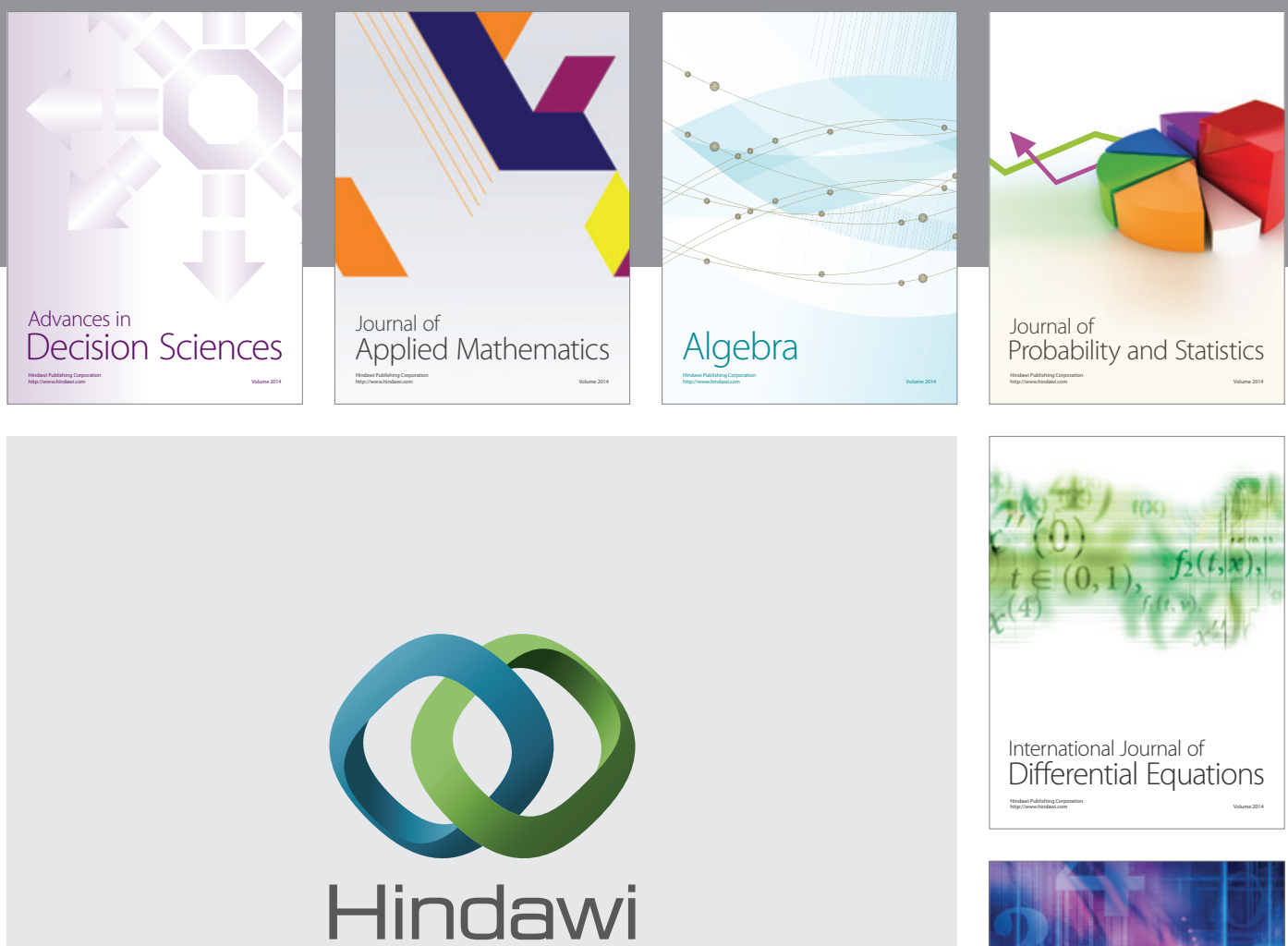

Submit your manuscripts at http://www.hindawi.com
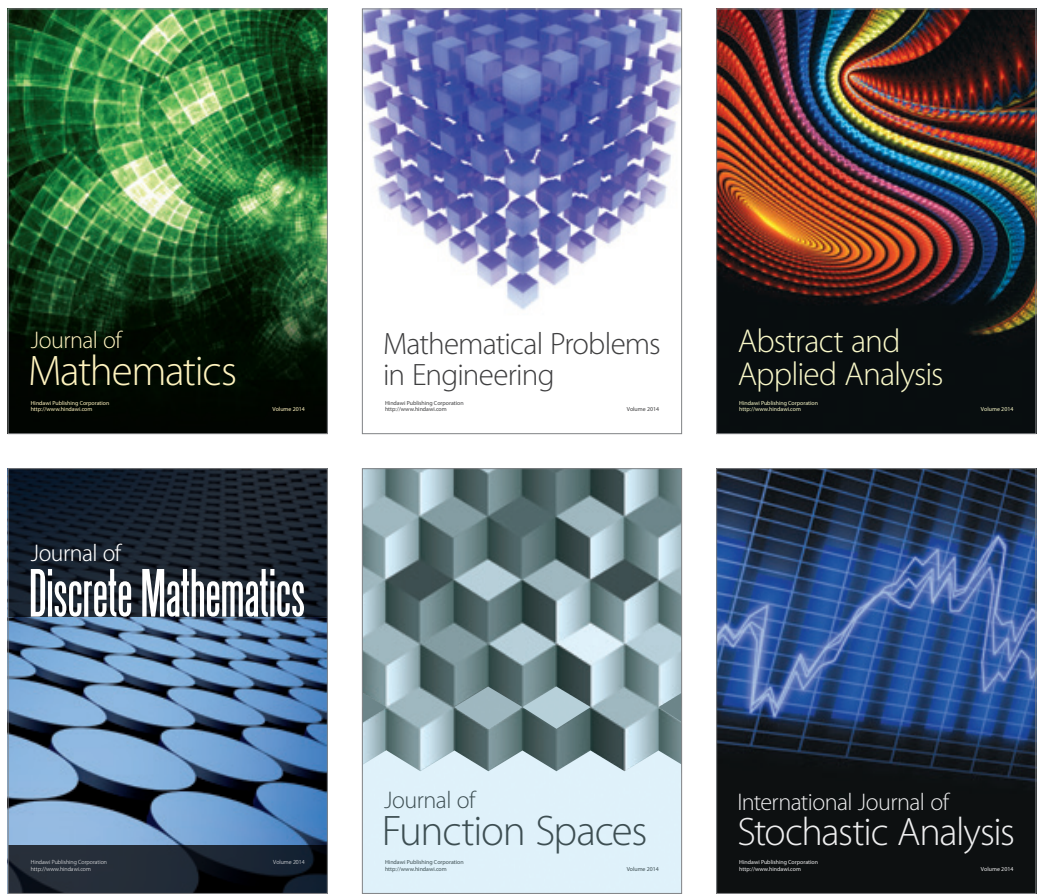

Journal of

Function Spaces

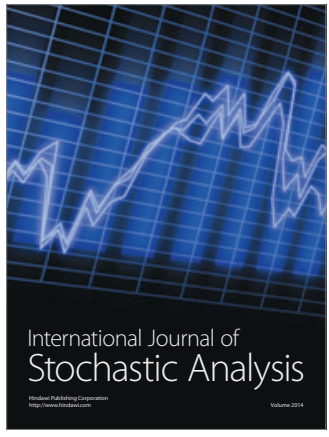

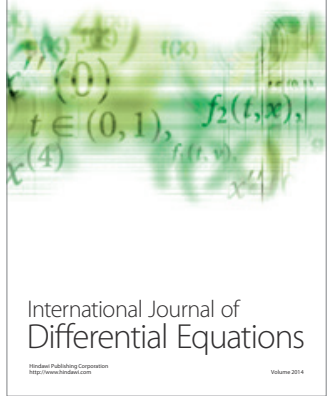
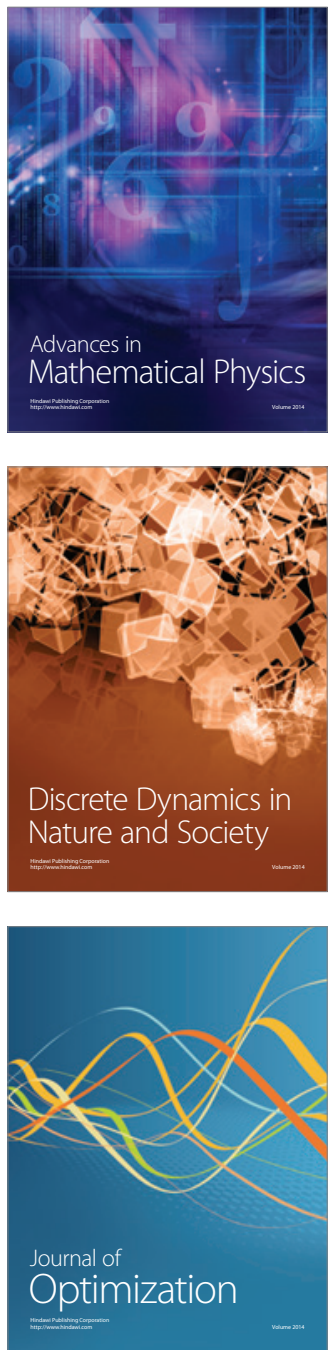